\title{
Correspondence
}

\section{Patients who need clozapine who refuse haematological monitoring}

Sir: One of my patients is being considered for clozapine but is refusing the regular blood tests needed for this and the question arises as to what to do.

The Mental Health Act Commission (1993) has issued advice on this point but this is not well known and I would like to quote it here.

"The Central Policy Committee determined that, since the blood monitoring was a condition of the licence for the use of the drug, if clozapine was authorised either by a Responsible Medical Officer or by the certificate of a Section 58 Appointed Doctor (SOAD), the administration of the medicine should include the authority for the necessary monitoring, and that it would be improper to withhold recommended and authorised treatment from detained non-consenting patients because of uncertainty about the authority to undertake blood tests.

The degree of resistance and its origins (e.g. religious objections) to the blood sampling should be taken into consideration by the RMO and SOAD when deciding whether to authorise the treatment.

The Central Policy Committee considered that whether the authority to secure a blood sample was in fact exercised by the RMO, when a detained patient actively refused to cooperate with the venepuncture, was a matter for the judgement of the RMO, in conjunction with the multidisciplinary team".

I would be very interested to hear whether anyone used venepuncture for non-consenting patients on clozapine and with what results.

Mental Health Act Commission (1993) Excerpt from Practice Note 1: Guidance on the Administration of Clozapine and Other Treatments requiring Blood Tests under the Proutsions of part $w$ of the Mental Health Act. Issued June 1993.

PhILIP STEAdMan, Sutton Hospital (St Helier NHS Trust), Cotswold Road, Sutton, Surrey SM2 5NF

\section{Administration of ECT by junior psychiatrists}

Sir: With regard to electroconvulsive treatment (ECT), worrying deficiencies in junior psychiatrists' knowledge, supervision and training have again been highlighted (Ramsay \& McPhillips, Psychiatric Bulletin, 1993, 17, 766775).

I was once contacted by a nervous senior house officer who had no idea how to administer ECT but was faced with giving it for the first time. She was unable to contact her senior colleagues who neglected to ensure she was adequately instructed on the procedure. I, as another junior officer with adequate training in ECT, had to instruct her where she should place the electrodes and how to set the machine. A seizure was successfully induced. She subse quently received supervision from her consultant on ECT.

If ECT was as invasive as psychosurgery, no consultant psychiatrists would dare allow their juniors to perform it without good knowledge that the juniors were competent to carry out the procedure. However, this is not the case and once the dial on the ECT machine is correctly set, the act of positioning electrodes on a human head and pressing a button could perhaps be done by a suitably trained chimpanzee. ECT training is not merely about learning dial setting electrode positioning and button pressing.

Undertaking work which is beyond one's competence constitutes negligence (Jones, 1992). Junior psychiatrists should appreciate that if they undertake to perform ECT without proper supervision and training from an approved body they risk being found liable to negligence if a treatment ended in a fatality.

Jones, M.A. (1992) Medical negligence. In Doctors, Patients and the Law (ed. Clare Dyer) pp. 11. Oxford: The Medical Protection Society in association with Blackwell Scientific Publications.

Russell D. Lutchman, Princess Alexandra Hospital, Harlow, Essex CM20 19X

\section{Measuring consumer satisfaction in a child psychiatry unit}

Sir: We were delighted to read the article by Elizabeth Walters emphasising the importance of 'asking the customer's opinion' in a child psychiatry context (Psychiatric Bulletin, 1993, 17, 661-662). Children's views are important but Dr Walters neglects to mention referrers (usually GPs) who are also consumers. We have completed a semi-structured self report survey of 
satisfaction of parents, referrers and children admitted to the residential child psychiatry unit at Guy's Hospital over a three year period.

Of the 50 children admitted to the unit, 25 carers, 22 children and 27 referrers responded. Of respondents $92 \%$ of carers, $82 \%$ of children and $85 \%$ of referrers found the admission helpful. Of the 13 responses that could be matched between carer, child and referrer, 12 agreed that admission was helpful.

Parents reported that the admission gave them greater understanding of the problem, increased children's confidence and gave parents a break while their child recovered. However, parents complained that other children were a bad influence on their child, the ward rules were too strict and some were concerned about insulation from the family.

Children reported that they now understood their problem, had more confidence, learnt to relax, could keep up their school work and made new friends. Children complained that they were homesick and unhappy, lacked privacy and that their particular problem was not solved.

Of referrers, $92 \%$ had received a summary. The intensive assessment had helped clarify problems and expert advice had helped parents cope, family functioning had improved and the children were more independent and confident. One referrer was unhappy as the problem still existed. Another felt that the mother's needs were not met.

As a consequence of these comments the unit has compiled a brochure and parents are involved more in the assessment and goal setting stages preceding admission.

KamaldeEP BHUI, SARAH DENNISON and TOM MORAN, Hector Cameron Ward, Guy's Hospital, London SE1 9RT

\section{Value of experience in child and family psychiatry}

Sir: I agree with Jan Hermsen's comments (Psychiatric Bulletin, 1993, 17, 628) on the value of experience in child and family psychiatry as part of general psychiatric training. It gives the trainee two specific advantages in treating adult patients: greater awareness of environmental and psychological influences on behaviour and emotion, and technical skills which make dealing with the families of adult patients less daunting and more therapeutically valuable. I often find it helpful to use my knowledge of systems theory, and techniques such as circular questioning, when faced by families attempting to cope with mental illness in an adult relative. It seems illogical to abandon a family approach when the patient reaches the age of 16 as childhood ex- periences and family environment have a crucial bearing on adult lives, including the development and experience of mental illness.

I was lucky enough to gain experience of child and family psychiatry at an early stage in my training, during my second senior house officer post. I found working as part of a multidisciplinary team that was determinedly (and appropriately) non-medical in outlook challenging but rewarding, and one that trainees are unlikely to encounter outside this field. Open discussion of emotions evoked by the nature of the work, for example as a result of over-identification with or projection of feelings onto children, fostered self-understanding. Personal psychotherapy was strongly encouraged as a further aid to self-knowledge and therapeutic effectiveness. (Indeed, I believe that local private psychotherapists made a reasonable living from seeing staff from the department!)

The benefits gained from working with children and families lead me to strongly support $\mathrm{Dr}$ Hermsen's suggestion that all trainees be encouraged to gain such experience but, in view of the shortage of posts, I fear that only a minority will be able to do so.

ADAM KIRBY, Windsor Clinic, Fazakerly Hospital, Liverpool L9 7AL

\section{Hypnosis and psychotherapy}

Sir: Interest in hypnosis has waxed and waned over the years. I have been on a course which guarantees to produce integral therapists. Almost anyone who pays the course fee, regardless of background, can subscribe to the course and at the end can declare him or herself a psychotherapist, adding a few initials after his or her name.

In a seminar given to senior registrars in child psychiatry I conveyed how I use hypnosis as a 'tool' for psychotherapy and my colleagues confirmed that hypnosis is a powerful medium to transmit psychotherapeutic interventions. However, none of the participants, consultants or senior registrars, knew of a body in the College that looks into its use and, if need be, produces guidelines on who should use it.

Such a powerful method may be left to people not qualified to deal with people in a vulnerable state, the case of most people seeking the help of a hypnotist. I wonder if the College can encourage colleagues who use hypnosis, and are interested in developing its use as a therapeutic tool, not as an end on its own, to get together and discuss potential uses and concerns.

A. K. DARWISH, Brynffynnon Child and Family Service, Pontypridd, Mid Glamorgan CF37 4DD 